\section{Mukopharmakon bei Bronchitis senkt die Komplikationsrate}

Das pflanzliche Mukopharmakon GeloMyrtol $^{\circledR}$ forte (Myrtol standardisiert) ist sowohl bei akuter als auch bei chronischer Bronchitis effektiv. Es wirkt sekretolytisch, mukolytisch und sekretomotorisch und steigert so die mukoziliäre Clearance. Die antioxidative, antiinflammatorische und antimikrobielle Wirkung vermindern die Schleimhautentzündung. Damit kann das Mukopharmakon einer Chronifizierung akuter Bronchitiden vorbeugen und die Anzahl der Exazerbationen bei chronischer Bronchitis, die den Verlust der Lungenfunktion beschleunigen, reduzieren.

Die Deutsche Gesellschaft für Pneumologie und Beatmungsmedizin sowie die Deutsche Atemliga empfehlen Myrtol neben anderen Mukopharmaka in ihrer Leitlinie zur Behandlung bei chronisch obstruktiver Bronchitis und bei Lungenemphysem.

\section{Weniger Antibiotikaverbrauch}

Mehrere Untersuchungen demonstrieren die Effektivität von Myrtol. In einer randomisierten, doppelblinden Multicenterstudie mit 676 Patienten mit akuter Bronchitis wurde das Präparat mit Cefuroxim, Ambroxol und Placebo verglichen. Über zwei Wochen gegeben war Myrtol in der Responderrate Placebo und rechnerisch auch den beiden aktiven Behandlungsarmen signifikant überlegen. In einer weiteren Studie erhielten 246 Patienten mit chronischer Bronchitis über sechs Monate entweder Myrtol (3x $300 \mathrm{mg} / \mathrm{d}$ ) oder Placebo. In der Verumgruppe konnte die für die Wintermonate typische Steigerung der Exazerbationsrate gesenkt werden. 49\% der Patienten, die mit dem Mukopharmakon behandelt wurden, benötigten keine Antibiotika, verglichen mit 38\% in der Kontrollgruppe.

Zudem verminderte die Therapie die Dauer der Antibiotikagabe: In der Myrtolgruppe erhielten $63 \%$ der Patienten bis zu sieben Tage Antibiotika, bei $77 \%$ der Patienten mit Placebo dauerte die Therapie länger. Husten und Auswurf wurden statistisch signifikant und klinisch relevant reduziert.

Fachpressekonferenz, Hamburg, 24.2.2010 Veranstalter: Pohl Boskamp

\title{
PNEUMONEWS
}

Herausgeber Klinik:

Prof. Dr. A. Gillissen, Kassel

Priv.-Doz. Dr. M. J. Kohlhäufl, Stuttgart-

Gerlingen

\section{Schriftleiter:}

Priv.-Doz. Dr. S. Budweiser, Regensburg (Beatmungsmedizin)

Priv.-Doz. Dr. G. Friedel, Stuttgart-Gerlingen (Thoraxchirurgie)

Prof. Dr. A. Ghofrani, Gießen, Dr. K. Gutjahr, Leipzig (Erkrankungen des Lungenkreislaufs)

Prof. Dr. A. Gillissen, Kassel (Asthma bronchiale)

Prof. Dr. M. Griese, München (Pädiatrische Pneumologie)

Prof. Dr. A. Günther, Priv.-Doz. Dr. Ph. Markart, Gießen (Interstitielle Lungenerkrankungen) Priv.-Doz. Dr. M. J. Kohlhäufl, Stuttgart-Gerlingen (Tumoren)

Dr. S. R. Ott, Bern (Infektiologie)

Prof. Dr. K. Rasche, Wuppertal, Prof. Dr. K.-H. Rühle, Hagen, Prof. Dr. H. Schäfer, Völklingen (Schlafmedizin)

Prof. Dr. G. Rohde, Maastricht/Niederlande (COPD, Emphysem)

Dr. K. Schultz, Bad Reichenhall (Rehabilitation)

Prof. Dr. M. Schwab, Prof. Dr. K. Mörike, Tübingen (Pharmakologische Therapie)

\section{Redaktion:}

Dr. rer. nat. Beate Schumacher (Chefredaktion, verantwortlich, Anschrift wie Verlag, beate.schumacher@springer.com)

Ute Kempf (ute.kempf@springer.com),Dr.Judith Neumaier (judith.neumaier@t-online.de), Sarah Pampel (Ärztin), Angelika Holzgreve (Assistenz Chefredaktion, angelika.holzgreve@springer. com)

\section{Verlag:}

Springer Medizin, ๑ Urban \& Vogel GmbH, Aschauer Str. 30, D-81549 München,

Tel.: o 89/20 30 43-13 00, Fax: -13 99, http://www.urban-vogel.de.

Springer Medizin ist Teil der Fachverlagsgruppe Springer Science+Business Media.

\section{Geschäftsführer:}

Harm van Maanen, Dr. med. Dirk Einecke, Ulrich Huber, Stephan Kröck, , Dr. med. Esther Wieland, Matthias Wissel.

\section{Anzeigenverkauf:}

Ines Spankau (Anzeigenleitung, -1339, verantwortlich, Anschrift wie Verlag), Anzeigenpreisliste Nr. 3 vom 1. Januar 2010

\section{Corporate Publishing:}

Dr. Ulrike Fortmüller (Leitung München, -1417)

\section{Herstellung:}

Frank Hartkopf (Leitung, -13 11); w\&Co MediaServices München GmbH \& Co KG (Layout); Dieter L. Adam, München (Titelbild)

\section{Vertrieb/Marketing Services:}

Frank Niemann (Leitung, -14 11)

\section{Abonnement:}

Die Zeitschrift erscheint $6 \times$ jährlich. Neue Bezugspreise ab 2010: Einzelheft $12 €$ inkl. der deutschen MwSt. zuzügl. 2,50 € Versandkosten; Jahresabo $75 €$; Studenten/Ärzte in Aus- und Weiterbildung $60 €$, jeweils zuzügl. Versand (Inland 19,50 €, Ausland $35 €$ ). Bestellungen nimmt der Verlag und jede Buchhandlung entgegen. Das Abonnement verlängert sich jeweils um ein Jahr, wenn es nicht zwei Monate vor Ende des Bezugszeitraums abbestellt wurde.

\section{Abonnentenservice:}

Tel.: o 62 21/3 45-43 04; Fax: o 62 21/3 45-42 29

Gesamtherstellung: Stürtz $\mathrm{GmbH}$, AlfredNobel-Str. 33, D-97080 Würzburg

Originalien: Zur Veröffentlichung kommen nur Arbeiten, die an anderer Stelle weder angeboten noch erschienen sind. Die Autoren sind verpflichtet zu prüfen, ob Urheberrechte Dritter berührt werden. Alle CME-Manuskripte werden von unabhängigen Experten begutachtet. Eine redaktionelle Bearbeitung bleibt vorbehalten. Autoren, die mit vollem Namen genannt sind, veröffentlichen ihre Beiträge in alleiniger Verantwortung.

Copyright: Der Verlag behält sich das ausschließliche Recht der Verbreitung, Übersetzung und jeglicher Wiedergabe auch von Teilen dieser Zeitschrift durch Nachdruck, Fotokopie, Mikrofilm, EDV-Verwertung, Funk- oder Fernsehaufzeichnung vor. Jede gewerblich hergestellte oder benutzte Fotokopie verpflichtet nach $\S 54$ (2) UrHG zur Gebührenzahlung an die VG Wort, Abt. Wissenschaft, Goethestr. 49, D-80336 München, von der die Modalitäten zu erfragen sind.

Rechtseinräumung der Autoren: Mit der Einsendung eines Manuskripts zur Veröffentlichung überträgt der Verfasser dem Verlag für den Fall der Annahme das Recht, das Manuskript geändert oder unverändert, ganz oder teilweise in Pneumo-News und anderen Publikationen der Fachverlagsgruppe, in den zugehörigen Onlinediensten, in Onlinedatenbanken Dritter und, soweit vereinbart, gegen Nachhonorar in Sonderdrucken für Industriekunden zu nutzen.

Hinweis: Die in dieser Zeitschrift angegebenen Dosierungen - vor allem von Neuzulassungen - sollten in jedem Fall mit dem Beipackzettel der verwendeten Medikamente verglichen werden.

ISSN 1865-5467

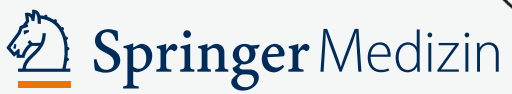

